CASE REPORT

\title{
Olaparib for metastatic breast cancer in a patient with a
} germline $P A L B 2$ variant

\author{
Sherko Kuemmel $\mathbb{D}^{1 凶}{ }^{凶}$, Hakima Harrach ${ }^{1}$, Rita K. Schmutzler ${ }^{2}$, Athina Kostara ${ }^{1}$, Katja Ziegler-Löhr ${ }^{3}$, Mark H. Dyson ${ }^{1}$, Ouafaa Chiari ${ }^{1}$ and \\ Mattea Reinisch (D)
}

There is a strong biologic rationale that poly(adenosine diphosphate-ribose) polymerase (PARP) inhibitors may benefit a broader range of metastatic breast cancer (MBC) patients than covered by current approvals, which require a germline $B R C A 1 / 2$ sequence variant affecting function. We report a patient with germline/somatic $B R C A 1 / 2$ wild-type $\mathrm{MBC}$, who had a dramatic response to the PARP inhibitor olaparib of at least 8 months' duration. The patient is a 37-year-old woman with recurrent, hormone receptorpositive, HER2-negative MBC that had progressed despite hormonal therapy and palbociclib. Sensitivity to olaparib was likely conferred by a germline sequence variant affecting function in PALB2 (exon 1, c.18G>T, p. $(=)$ )). This case documenting activity of olaparib monotherapy in germline/somatic BRCA1/2 wild-type MBC illustrates that the clinical potential of PARP inhibition in MBC extends beyond currently approved indications to additional patients whose tumors have (epi)genetic changes affecting homologous recombination repair.

npj Breast Cancer (2020)6:31; https://doi.org/10.1038/s41523-020-00174-9

\section{INTRODUCTION}

The poly(adenosine diphosphate-ribose) polymerase (PARP) inhibitors olaparib and talazoparib are approved for treatment of hormone receptor (HR)-positive or triple-negative advanced breast cancer in patients with germline variants affecting function of breast cancer susceptibility genes 1 or 2 (BRCA1/2). The approved indications derive from the eligibility criteria for the randomized Phase 3 trials of these agents versus a variety of standard chemotherapy options in this setting, which required central verification of germline $B R C A 1 / 2$ sequence variation ${ }^{1,2}$. Selection of this target population was based on the homologous recombination deficiency (HRD) caused by loss of functional BRCA1/2, which renders cancer cells unable to accurately repair the double-strand DNA breaks induced by PARP inhibitors ${ }^{3}$. Nonetheless, according to this biologic rationale, the spectrum of breast cancers with the potential to respond to PARP inhibitors may extend beyond patients with germline $B R C A 1 / 2$ sequence variants to include those with germline or somatic sequence variants, or epigenetic inactivation, of these or other genes involved in maintenance of genomic integrity ${ }^{3}$.

We present the case of a patient with metastatic breast cancer (MBC) without a germline or somatic BRCA1/2 sequence variant, but with a germline sequence variant affecting function in partner and localizer of BRCA2 (PALB2), who exhibited a dramatic response to olaparib. This case illustrates the clinical potential of PARP inhibitors in MBC outside currently approved indications.

\section{RESULTS}

Case history and presentation

In October 2013, a 31-year-old woman was diagnosed with estrogen receptor-positive (70\%), progesterone receptor-positive (15\%), human epidermal growth factor receptor-2 (HER2)-negative, high grade (G3) invasive cancer of the right breast (pT1c, pN1a, M0; Ki-67 index 20\%), with concurrent ductal carcinoma in situ (DCIS). Primary treatment was subcutaneous right mastectomy with implant-based reconstruction, adjuvant chemotherapy (epirubicin/cyclophosphamide and docetaxel), irradiation of the affected breast and supra- and infraclavicular nodes, and adjuvant tamoxifen, which was discontinued after 8 weeks due to a major depressive episode. Thereafter, the patient remained relapse free for 2 years, until positron emission tomography (PET)/computed tomography (CT) revealed local recurrence in the thoracic wall with mediastinal nodal involvement with no other distant metastases. Salvage mediastinal lymphadenectomy was performed, but the patient initially declined further systemic therapy. However, 14 months later, local recurrence, distant bone and liver metastasis, and deteriorating clinical condition led to initiation of palliative therapy with letrozole, a gonadotropin-releasing hormone analog, palbociclib, and denosumab. Partial radiologic response (per Response Evaluation Criteria in Solid Tumors (RECIST) v1.1) was attained under therapy until PET/CT after 11 months indicated progression of bone metastases and a new pericardial lesion.

\section{Genetic analysis}

The patient reported no family history of breast cancer at initial diagnosis and tested negative for germline sequence variants in $B R C A 1, B R C A 2, C H E K 2$, and RAD51C. However, subsequent genetic analysis by next-generation sequencing (NGS) identified a germline sequence variant in PALB2 (exon 1, c.18G $>\mathrm{T}, \mathrm{p} .(=)$ ) of class IV (i.e., likely pathogenic). Although the patient's mother was diagnosed with DCIS during the course of disease, genetic profiling of the mother indicated that the patient's germline variant had in fact been paternally inherited. NGS analysis of mediastinal nodal tumor tissue acquired at relapse indicated a microsatellite-stable tumor with low mutational burden (4 mut/ $\mathrm{Mb})$, ARID1A mutation (Q1409*), and amplification of RAD21 and $R P O R$, but yielded no clinically actionable findings. We had limited therapeutic options in this setting due to patient refusal of

${ }^{1}$ Interdisciplinary Breast Unit, Kliniken Essen-Mitte, Essen, Germany. ${ }^{2}$ Center for Familial Breast and Ovarian Cancer, Center for Integrated Oncology (CIO), University of Cologne, Faculty of Medicine and University Hospital Cologne, Cologne, Germany. ${ }^{3}$ Schwerpunktpraxis für Gynäkologische Onkologie, Cologne, Germany. ${ }^{凶}$ email: s.kuemmel@kem-med.com 


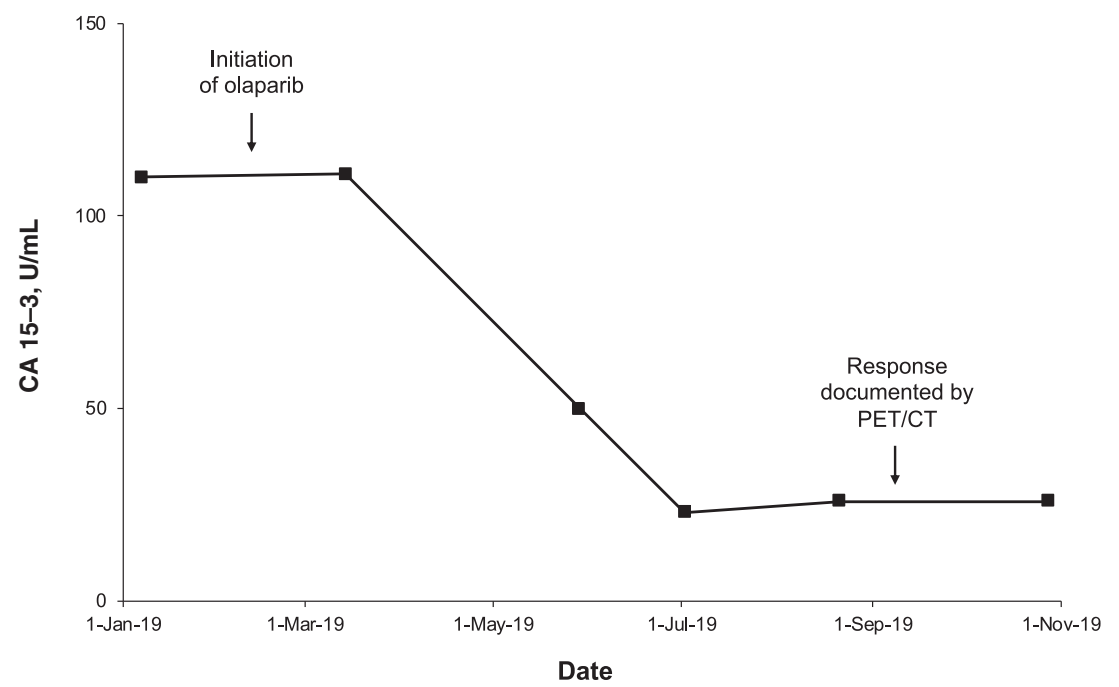

Fig. 1 Tumor marker response to olaparib. Compared with pretreatment levels, there was a marked reduction in circulating CA 15-3 at 3 months after initiation of olaparib, which was deepened and sustained after 8 months of treatment.

palliative chemotherapy. Given the well-established efficacy of PARP inhibitors in patients with germline BRCA1/2 sequence variants ${ }^{1,2}$ and the phenotypic similarities between PALB2-mutant and BRCA1/2-mutant cells ${ }^{4}$, we reasoned that olaparib could provide clinical benefit for this patient with a germline PALB2 variant and HR-positive, HER2-negative MBC that had progressed despite endocrine and cyclin-dependent kinase-4/6 inhibitor therapy.

\section{Management}

In February 2019, off-label treatment with olaparib was initiated at a dose of $600 \mathrm{mg} /$ day (two 150-mg tablets twice daily), alongside continuing therapy with denosumab. The olaparib dose was reduced to $200 \mathrm{mg} /$ day (one 100-mg tablet twice daily) after 3 weeks to manage side effects of nausea, vomiting, and fatigue that had not responded to supportive therapy. Within a few weeks, the patient reported an improvement in well-being, with a reduction in pain, increased appetite, and enhanced quality of life. There was a substantial reduction in tumor marker CA 15-3 by month 3 that was sustained after 8 months of therapy (Fig. 1). Based on patient preference, the first restaging was conducted 7 months after initiation of olaparib by PET/CT (Fig. 2). The scan demonstrated significant regression of all lesions, corresponding to a partial response per RECIST v1.1. The only remaining PETpositive lesions consisted of a left inguinal lymph node and low residual activity of skeletal metastases in the thoracic spine and the right sacrum and ilium. After 8 months of therapy, the patient remains in good clinical condition, continues to receive olaparib, and will be followed-up with regular tumor marker assessment and $\mathrm{PET} / \mathrm{CT}$.

\section{DISCUSSION}

This case report documents response to olaparib monotherapy in a patient with $M B C$ with a sequence variant affecting function in PALB2. Like BRCA1/2, PALB2 encodes a homologous recombination repair protein whose loss of function produces synthetic lethality in combination with PARP inhibition ${ }^{4}$. Sensitivity to olaparib may, therefore, be attributable to this PALB2 variant ${ }^{5,6}$. In addition, the tumor showed a loss-of-function alteration in the chromatin remodeling complex subunit $A R I D 1 A$, which also leads to defective double-strand break repair and vulnerability to PARP inhibition ${ }^{7}$. While variants in PALB2 and ARID1A are implicated in only a small proportion of breast cancers $(0.5-1 \% \text { and } 4 \% \text {, respectively })^{8-10}$, this case provides proof-of-concept of the therapeutic potential of PARP inhibitors in a wider range of MBC patients than covered by current approvals. In a large series of unselected breast cancers, $16 \%$ of tumors had inherited or acquired driver sequence variants across a panel of homologous recombination repair genes, including BRCA1/2, PALB2, ARID1A, ATM, CHEK2, and BAP $1^{10}$. A higher proportion of breast cancers ( $>20 \%$ overall; up to $70 \%$ in triple-negative disease) test positive in assays that detect genomic scars characteristic of HRD, irrespective of the underlying (epi) genetic mechanism ${ }^{11,12}$.

Our patient showed a duration of response of at least 8 months (ongoing at the time of writing), which compares favorably with the median progression-free survival of 7.0 months with olaparib in the Phase 3 OlympiaD trial ${ }^{1,13}$. That study also provided evidence of overall survival benefit versus chemotherapy among patients who, like the present case, had not received prior chemotherapy for metastatic disease ${ }^{13}$. While the published Phase $2 / 3$ olaparib and talazoparib MBC trials have focused on patients with germline $B R C A 1 / 2$ sequence variants ${ }^{1,2,14,15}$, several observations suggest a broader range of patients with $M B C$ could benefit. For example, complete response was previously reported in a patient with wild-type germline $B R C A 1 / 2$ status in a Phase 1 olaparib study ${ }^{16}$. However, the response cannot be unambiguously attributed to olaparib due to the coadministration of paclitaxel and, as far as we are aware, no further germline or somatic genetic profiling of this patient has been reported. Clinical activity of olaparib monotherapy has also been documented in a patient with triple-negative $\mathrm{MBC}$ with brain metastasis and a somatic sequence variant in $B R C A 1^{17}$. Results reported in abstract form describe response to talazoparib in two patients with wildtype germline/somatic BRCA1/2 MBC and a germline PALB2 variant ${ }^{18}$. Recently, Grellety et al. also described a marked response to olaparib in a patient with HR-positive, HER2-negative, $B R C A 1 / 2$ wild-type $M B C$, and a germline variant in PALB2 different from the one detected in the present case, as well as a second PALB2 mutation in the tumor ${ }^{19}$. In addition, subgroup analyses of Phase 2 trials provide evidence for the efficacy of PARP inhibitors in combination with neoadjuvant chemotherapy in early breast cancer with wild-type germline BRCA1/2 (I-SPY 2$)^{20}$ or wild-type germline and somatic BRCA1/2 and HRD (GeparOLA) ${ }^{21}$. Beyond breast cancer, trials in advanced ovarian cancer and metastatic castration-resistant prostate cancer have demonstrated efficacy of various PARP inhibitors in populations other than carriers of germline sequence variants in $B R C A 1 / 2$, including somatic $B R C A 1 / 2$ variant-associated cancers, and tumors with or without $\mathrm{HRD}^{22,23}$. 

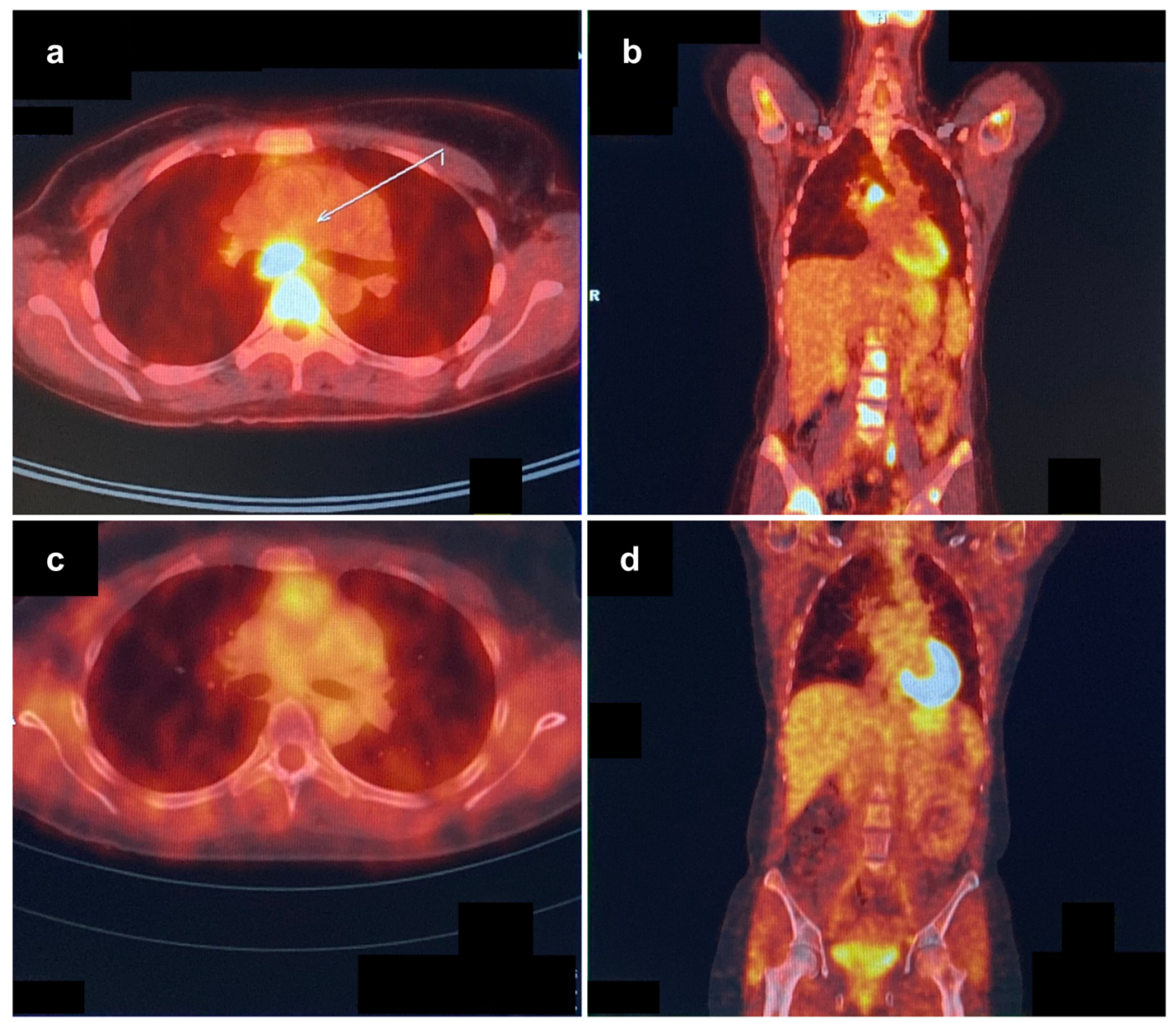

Fig. 2 Radiologic response to olaparib. Axial (a) and coronal (b) PET/CT images acquired prior to initiation of olaparib show hypermetabolic mediastinal lymph nodes and multifocal bony attachment in the spine and pelvis. After 7 months of olaparib therapy, axial (c) and coronal (d) $\mathrm{PET} / \mathrm{CT}$ images indicate significant remission of lymph node and bone metastases.

Female heterozygous carriers of sequence variants affecting function in PALB2 have a cumulative breast cancer risk of $14 \%$ by age 50 years and $35 \%$ by age 70 years ${ }^{5}$. The current case is consistent with the typical presentation of breast cancers in PALB2 sequence variant carriers, which frequently show high nuclear grade and mitotic index, with estrogen receptorpositivity (53-74\%; although triple-negative disease is significantly overrepresented among carriers of pathogenic PALB2 variants) ${ }^{5,24,25}$. The observed PALB2 variant (exon 1, c.18G>T, p. $(=))$ is a synonymous change that leads to abnormal transcript splicing and loss of function via premature termination of translation $^{26}$. Given the poor prognostic impact of germline PALB2 sequence variants and limited evidence regarding systemic therapy in this subgroup ${ }^{25}$, the marked response in the present case is particularly noteworthy.

In conclusion, this case report documenting response to olaparib of at least 8 eight months' duration in a patient with progressive $M B C$ lacking germline or somatic $B R C A 1 / 2$ sequence variants illustrates the clinical potential of olaparib monotherapy in $\mathrm{MBC}$ beyond currently approved indications. Taken together with the biologic rationale for synthetic lethality of PARP inhibition in tumors with $\mathrm{HRD}^{3}$, these data suggest that additional patients with relapsed and/or metastatic disease could benefit from this therapeutic strategy. We keenly await the results of ongoing clinical trials of PARP inhibitors in broader MBC populations (e.g., NCT03344965, NCT02401347, and NCT02029001), including patients with germline or somatic sequence variants in other HRD driver genes or HRD-high status in genomic assays.

\section{METHODS}

Ethics statement

Off-label treatment with olaparib in this patient with MBC lacking germline $B R C A 1 / 2$ sequence variants affecting function was provided as compassionate use in the palliative setting in line with national regulations and did not require ethics committee approval. The patient provided written informed consent prior to initiation of treatment. Written informed patient consent was also obtained for publication of the data contained in this case report.

\section{Genetic analyses}

Germline genetic analysis was conducted by NGS using the TruRisk ${ }^{\circ}$ Version 2 gene panel, which includes 15 core genes: ATM, BRCA1, BRCA2, BRIP1, CDH1, CHEK2, MLH1, MSH2, MSH6, PALB2, PMS2, PTEN, RAD51C, RAD51D, and TP53. Genomic DNA was extracted from blood samples, enriched for target sequences with the SureSelect ${ }^{\mathrm{XT}}$ kit (Agilent Technologies, Inc., Santa Clara, CA, USA), and directly sequenced using the NextSeq platform (Illumina, Inc., San Diego, CA, USA). For tumor genetic analysis, DNA was extracted from formalin-fixed, paraffinembedded tumor samples and analyzed using a commercial NGS assay (FoundationOne ${ }^{\oplus} \mathrm{CDx}$, Foundation Medicine, Inc., Cambridge, MA, USA).

\section{Reporting summary}

Further information on experimental design is available in the Nature Research Reporting Summary linked to this article.

\section{DATA AVAILABILITY}

The data generated and analyzed in this study are described in the following metadata record: https://doi.org/10.6084/m9.figshare.12370346 ${ }^{27}$. The tumor DNA sequencing data have been deposited in NCBI Sequence Read Archive and are 
available via accession: https://identifiers.org/ncbi/bioproject:PRJNA635377 ${ }^{28}$. The tumor DNA NGS analysis is contained in the spreadsheet Tumor DNA analysis.xlsx, which is included with this data record ${ }^{27}$; this file summarizes the mutant allele frequency and copy number variations that were detected by NGS. The germline DNA sequencing cannot be shared due to German data protection law considerations designed to safeguard patient anonymity. However, the authors confirm there were no other rare germline variants detected besides the reported PALB2 variant, which has previously been described and is available in dbSNP under this accession: https://identifiers.org/dbsnp:rs587782462 ${ }^{29}$. The data underlying Fig. 1 in the related manuscript are contained in the spreadsheet CA 15-3.xlsx, included with this data record $^{27}$.

Received: 5 February 2020; Accepted: 23 June 2020; Published online: 24 July 2020

\section{REFERENCES}

1. Robson, M. et al. Olaparib for metastatic breast cancer in patients with a germline BRCA mutation. N. Engl. J. Med. 377, 523-533 (2017).

2. Litton, J. K. et al. Talazoparib in patients with advanced breast cancer and a germline BRCA mutation. N. Engl. J. Med. 379, 753-763 (2018).

3. Cerrato, A., Morra, F. \& Celetti, A. Use of poly ADP-ribose polymerase [PARP] inhibitors in cancer cells bearing DDR defects: the rationale for their inclusion in the clinic. J. Exp. Clin. Cancer Res. 35, 179 (2016).

4. Buisson, R. et al. Cooperation of breast cancer proteins PALB2 and piccolo BRCA2 in stimulating homologous recombination. Nat. Struct. Mol. Biol. 17, 1247-1254 (2010).

5. Antoniou, A. C. et al. Breast-cancer risk in families with mutations in PALB2. N. Engl. J. Med. 371, 497-506 (2014).

6. Rahman, N. et al. PALB2, which encodes a BRCA2-interacting protein, is a breast cancer susceptibility gene. Nat. Genet. 39, 165-167 (2007).

7. Shen, J. et al. ARID1A deficiency impairs the DNA damage checkpoint and sensitizes cells to PARP inhibitors. Cancer Discov. 5, 752-767 (2015).

8. Dansonka-Mieszkowska, A. et al. A novel germline PALB2 deletion in Polish breast and ovarian cancer patients. BMC Med. Genet. 11, 20 (2010).

9. Erkko, $\mathrm{H}$. et al. A recurrent mutation in PALB2 in Finnish cancer families. Nature 446, 316-319 (2007).

10. Heeke, A. L. et al. Prevalence of homologous recombination-related gene mutations across multiple cancer types. JCO Precis. Oncol. 2, (2018).

11. Davies, $H$. et al. HRDetect is a predictor of BRCA1 and BRCA2 deficiency based on mutational signatures. Nat. Med. 23, 517-525 (2017).

12. Loibl, S. et al. Survival analysis of carboplatin added to an anthracycline/taxanebased neoadjuvant chemotherapy and HRD score as predictor of response-final results from GeparSixto. Ann. Oncol. 29, 2341-2347 (2018).

13. Robson, M. E. et al. OlympiAD final overall survival and tolerability results: olaparib versus chemotherapy treatment of physician's choice in patients with a germline BRCA mutation and HER2-negative metastatic breast cancer. Ann. Oncol. 30, 558-566 (2019).

14. Kaufman, B. et al. Olaparib monotherapy in patients with advanced cancer and a germline BRCA1/2 mutation. J. Clin. Oncol. 33, 244-250 (2015).

15. Turner, N. C. et al. A Phase II study of talazoparib after platinum or cytotoxic nonplatinum regimens in patients with advanced breast cancer and germline BRCA1/2 mutations (ABRAZO). Clin. Cancer Res. 25, 2717-2724 (2019).

16. Dent, R. A. et al. Phase I trial of the oral PARP inhibitor olaparib in combination with paclitaxel for first- or second-line treatment of patients with metastatic triple-negative breast cancer. Breast Cancer Res. 15, R88 (2013).

17. Pascual, T. et al. Significant clinical activity of olaparib in a somatic BRCA1mutated triple-negative breast cancer with brain metastasis. JCO Precis. Oncol. 3, (2019).

18. Gruber, J. J. et al. Talazoparib beyond BRCA: a phase II trial of talazoparib monotherapy in BRCA1 and BRCA2 wild-type patients with advanced HER2negative breast cancer or other solid tumors with a mutation in homologous recombination (HR) pathway genes. J. Clin. Oncol. 37, 3006 (2019).

19. Grellety, T. et al. Dramatic response to PARP inhibition in a PALB2-mutated breast cancer: moving beyond BRCA. Ann. Oncol. https://doi.org/10.1016/j. annonc.2020.03.283 (2020).

20. Wolf, D. M. et al. DNA repair deficiency biomarkers and the 70-gene ultra-high risk signature as predictors of veliparib/carboplatin response in the I-SPY 2 breast cancer trial. NPJ Breast Cancer 3, 31 (2017).

21. Fasching, P. A. et al. GeparOLA: a randomized phase II trial to assess the efficacy of paclitaxel and olaparib in comparison to paclitaxel/carboplatin followed by epirubicin/cyclophosphamide as neoadjuvant chemotherapy in patients (pts) with HER2-negative early breast cancer (BC) and homologous recombination deficiency (HRD). J. Clin. Oncol. 37, 506 (2019).

22. Moore, K. et al. Maintenance olaparib in patients with newly diagnosed advanced ovarian cancer. N. Engl. J. Med. 379, 2495-2505 (2018).

23. Mateo, J. et al. DNA-repair defects and olaparib in metastatic prostate cancer. $N$. Engl. J. Med. 373, 1697-1708 (2015).

24. Heikkinen, T. et al. The breast cancer susceptibility mutation PALB2 1592delT is associated with an aggressive tumor phenotype. Clin. Cancer Res. 15, 3214-3222 (2009).

25. Cybulski, C. et al. Clinical outcomes in women with breast cancer and a PALB2 mutation: a prospective cohort analysis. Lancet Oncol. 16, 638-644 (2015).

26. Yang, C. et al. A synonymous germline variant PALB2 c.18G>T (p.Gly6=) disrupts normal splicing in a family with pancreatic and breast cancers. Breast Cancer Res. Treat. 173, 79-86 (2019).

27. Kuemmel, S. et al. Metadata record for the manuscript: olaparib for metastatic breast cancer in a patient with a germline PALB2 variant. (figshare, 2020). https:// doi.org/10.6084/m9.figshare.12370346.

28. ID 635377 BioProject. NCBI Sequence Read Archive. https://identifiers.org/ncbi/ bioproject:PRJNA635377 (2020).

29. rs587782462 RefSNP Report. dbSNP. https://identifiers.org/dbsnp:rs587782462 (2020).

\section{AUTHOR CONTRIBUTIONS}

S.K., H.H., O.C., and M.R. contributed to data collection, interpretation, literature searching, and manuscript drafting. R.K.S. contributed to data collection, interpretation, and critical review of the manuscript. A.K. and K.Z.-L. contributed to data collection and critical review of the manuscript. M.H.D. contributed to interpretation, literature searching, and manuscript drafting. All authors approved the final manuscript draft.

\section{COMPETING INTERESTS}

S.K. reports receiving personal fees from Roche, Genomic Health, Novartis, Amgen, Celgene, Daiichi Sankyo, Astra Zeneca, Somatex, MSD, Pfizer, Puma Biotechnology, PFM Medical and Lilly, and nonfinancial support from Roche, Daiichi Sankyo, Somatex and Sonoscape (outside the submitted work). A.K. reports receiving personal fees from Astra Zeneca and nonfinancial support from Tesaro and MSD (outside the submitted work). M.H.D. reports receiving personal fees from Boehringer Ingelheim, Merck, Novartis, EUSA Pharma, AbbVie, Janssen, Biogen, Menarini, and Norgine (outside the submitted work). M.R. reports receiving personal fees from Novartis, Lilly, Roche, Pfizer, Astra Zeneca, MSD and Somatex, and travel grants from Novartis, Pfizer and Celgene. Other authors report no competing interests.

\section{ADDITIONAL INFORMATION}

Supplementary information is available for this paper at https://doi.org/10.1038/ s41523-020-00174-9.

Correspondence and requests for materials should be addressed to S.K.

Reprints and permission information is available at http://www.nature.com/ reprints

Publisher's note Springer Nature remains neutral with regard to jurisdictional claims in published maps and institutional affiliations.

Open Access This article is licensed under a Creative Commons Attribution 4.0 International License, which permits use, sharing, adaptation, distribution and reproduction in any medium or format, as long as you give appropriate credit to the original author(s) and the source, provide a link to the Creative Commons license, and indicate if changes were made. The images or other third party material in this article are included in the article's Creative Commons license, unless indicated otherwise in a credit line to the material. If material is not included in the article's Creative Commons license and your intended use is not permitted by statutory regulation or exceeds the permitted use, you will need to obtain permission directly from the copyright holder. To view a copy of this license, visit http://creativecommons. org/licenses/by/4.0/.

(c) The Author(s) 2020 